\title{
STEREOMETRIC MODELLING
}

Pietro Grimaldi

Laboratorio di Fotogrammetria Architettonica del Politecnico di Bari, Italy

fotogrammetria@gmail.com

\section{Commission V/2}

KEY WORDS: Cultural Heritage, Modelling, Photogrammetry, Restitution, Stereoscopic On-line, Teaching, Virtual Reality.

\begin{abstract}
:
These mandatory guidelines are provided for preparation of papers accepted for publication in the series of Volumes of The The stereometric modelling means modelling achieved with :

- the use of a pair of virtual cameras, with parallel axes and positioned at a mutual distance average of $1 / 10$ of the distance camera-object (in practice the realization and use of a stereometric camera in the modeling program);

- the shot visualization in two distinct windows

- the stereoscopic viewing of the shot while modelling.
\end{abstract}

Since the definition of "3D vision" is inaccurately referred to as the simple perspective of an object, it is required to add the word stereo so that "3D stereo vision " shall stand for "three-dimensional view" and ,therefore, measure the width, height and depth of the surveyed image.

Thanks to the development of a stereo metric model, either real or virtual, through the "materialization", either real or virtual, of the optical-stereo metric model made visible with a stereoscope.

It is feasible a continuous on line updating of the cultural heritage with the help of photogrammetry and stereometric modelling. The catalogue of the Architectonic Photogrammetry Laboratory of Politecnico di Bari is available on line at:

http://rappresentazione.stereofot.it:591/StereoFot/FMPro?-db=StereoFot.fp5\&-lay=Scheda\&format $=$ cerca.htm \&-view

\section{AIMS}

The stereometric modelling means modelling achieved with :

- the use of a pair of virtual cameras, with parallel axes and positioned at a mutual distance average of $1 / 10$ of the distance camera-object (in practice the realization and use of a stereometric camera in the modeling program);

- the shot visualization in two distinct windows

- the stereoscopic viewing of the shot while modelling

In addition to both the basic measuring process and two- dimensional graph drawing,

the concept of photogrammetric restitution allows to develop a stereometric model, either real or virtual, through the "materialization", either real or virtual, of the opticalstereometric model made visible with stereoscope.

\section{METHOD}

Since the definition of "3D vision" is inaccurately referred to as the simple perspective of an object, it is required to add the word stereo so that "3D stereo vision " shall stand for "three- dimensional view" and ,therefore, measure the width, height and depth of the surveyed image.

After the perspective restitution of which the limits are easily detected in the "accelerated perspective", an increasing number of software systems have led people with little knowledge in Informatics or Photogrammetry to believe that it is possible to obtain the 
metric data from a single photogram taken with a conventional camera.

The mere plane representation makes the object modelling unimaginable as well as a blindfold craftsman at work.

\section{Tools used}

The modeling stereometric simply requires:

- the application of a stereoscope on the monitor;

- the creation of a virtual stereocamera, namely the use of two cameras side by side in the modeling program;

- the display of images taken in two separate windows, so that they are viewed separately through the stereoscope;

- taining that is very different from that required for learning to play the piano.

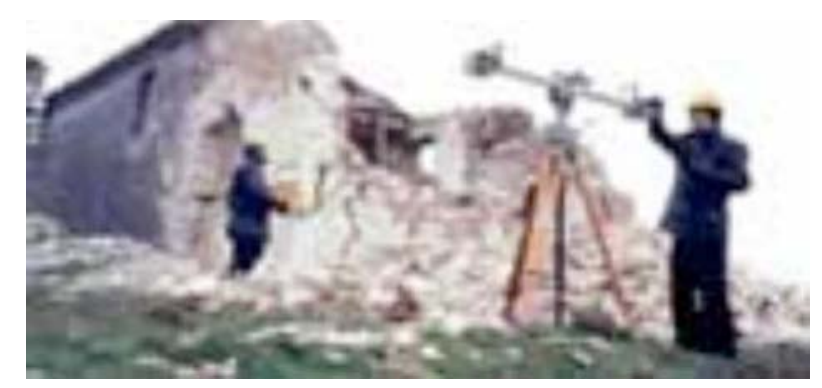

In the experimental phase of the modeling

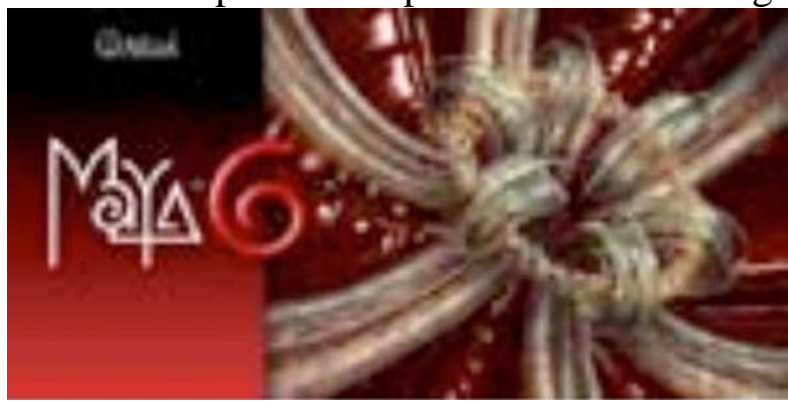

stereometric were used:

A) Macintosh computer with PowerPC G5 Dual 2GHz, 1 GB of memory, dual screens and a mirror stereoscope Wild ST4;

B) Maya 6.0, for modeling. The program allows the observation of the model from different points of view and, therefore, the model can be photographed stereoscopically;

C) Stereometric camera WILD C120 with the following characteristics:

based m.1, 20, $60 \mathrm{~mm}$ focal length., Image size $60 \mathrm{~mm}$ (vertical) x $80 \mathrm{~mm}$ (horizontal), Vertical shift $10 \mathrm{~mm}$., horizontal angle $64^{\circ}$, angle of asymmetrical vertical $\left(17^{\circ},+32^{\circ}\right)$, distance between the reference mark $75 \mathrm{~mm}$ (horizontal), height of the ground varies from $1.5 \mathrm{~m}$ to $2.5 \mathrm{~m}$., possible inclination to the horizontal plane $10^{\circ}, 30^{\circ}, 60^{\circ}, 80^{\circ}, 90^{\circ}$.

D) StereoFot ${ }^{\circledR}$ program, available on the Internet, which allows

consult the catalog photogrammetric

(http://rilievo.stereofot.it:591/StereoFot/FM Pro?- $\mathrm{db}=$ StereoFot.fp5\&-lay=Scheda\&format $=$ cercaST24.htm\&- view )

to obtain the stereoscopic observations become and to achieve the photogrammetric restitution of the frames made with the stereocamera WILD C120.

- $\quad$ image size 6 × $8 \mathrm{~cm}$;

- principal axes parallel;

- the basis of recovery equal to m. 1.20;

- principal distance of $63.68 \mathrm{~mm}$, photographic camera left created in Maya;

- principal distance of $63.75 \mathrm{~mm}$, photographic camera right created in Maya;

The right and left frames were imported into the corresponding cameras created in Maya, and attached to them.

In order to verify the accuracy have been created and placed four inverted cones planimetrically at points A $(0.60,6.00)$, B ($9.40,20.00), C(0.60,20.00)$ and D (10.60, 20.00). The vertices of the cones are perfectly coincident with the four existing designs in the field test, as it is possible to detect frames below.

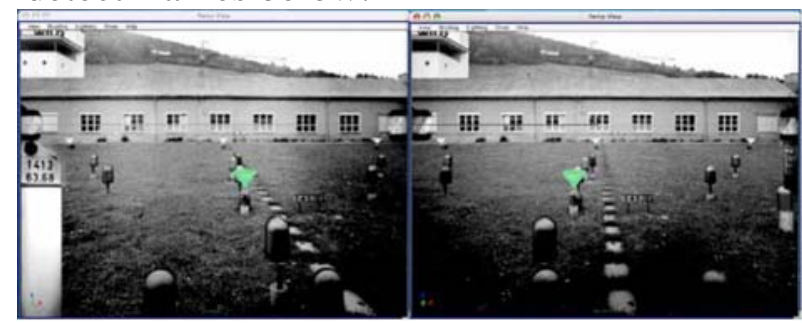

A practical application of what has been applied until now exhibited in the representation of the relief of the Cathedral of stereophotogrammetric Acerenza (PZ). The survey of Acerenza Cathedral (Church of SS. Maria Assunta) was executed 
December 19, 1980 by the Photogrammetry Unit of the Traffic Police in Bari, following the earthquake in Basilicata, in November of that year. The survey covers the interior of the church is composed of n.38 shooting stations, arranged according to the diagram below

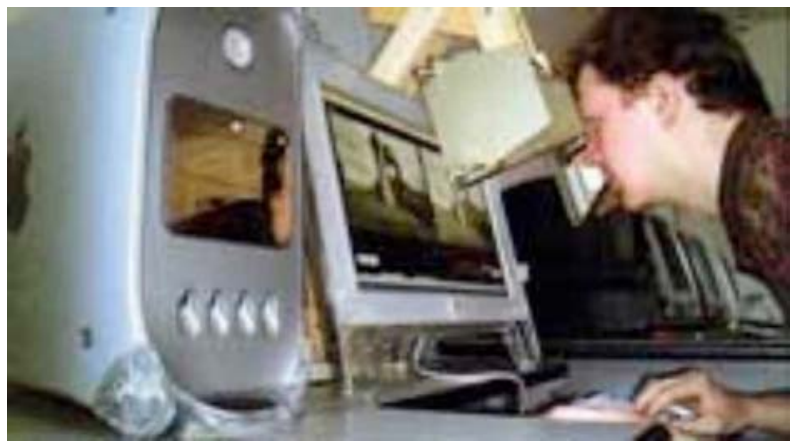

(http://rappresentazione. stereofot. it: 591/StereoFot/FMPro? - db=stereofot.fp5\&format $=$ dettagliost 24. htm \&lay $=$ scheda\&luogo $=$ Acerenz $a \&-\max =5 \&-$ recid $=102 \&$ - find $=$ )
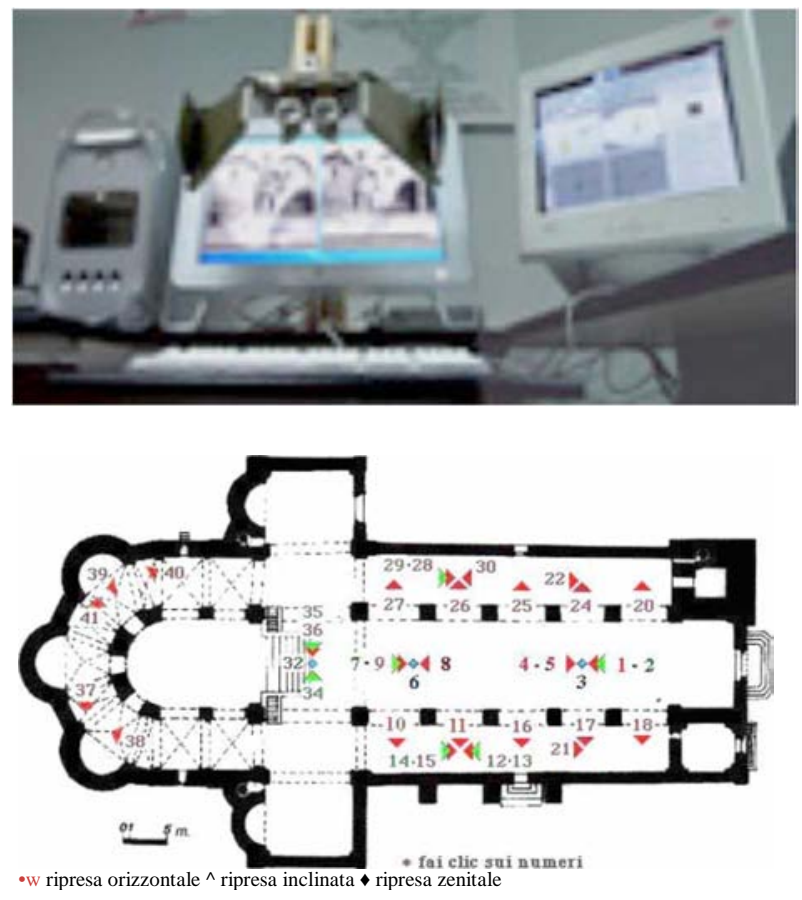

\section{Import frames}

For criteria used were the frames of the field test of the Wild C120.

For stereo recording was created a couple of cameras, together as a group and having the same characteristics of the two chambers of C120, namely:

\section{Import survey}

The modeling program used in this experiment, is Maya 6.0. To import the survey, create many "scenes" as the number of survey stations. In a second step, taking care of the overlap of common objects, you can import the resulting models in a single scene.

For each station, the operations performed are the following:

1. choice of the reference system with the "z" axis vertical;

2. creation of a photographic camera with the following characteristics:

- The name "sinistra1";

- Position in the origin of the reference frame $(0,0,0)$;
- Optical axis horizontal and coincident with the axis "y";

- Principal distance $63.47 \mathrm{~mm}$;

- $80 \mathrm{~mm}$ image format. (3149 inch) x $60 \mathrm{~mm}$. (2362 inch);

- angle imaging 64.43;

- Background, as "imageSinistra1", the left frame (No. 1987) of the first - resort (digitized in 800 x 600 pixels with 72 dpi resolution), - decentralized than $10 \mathrm{~cm}$. (0393 inch);

3. duplication of the photographic camera "sinistra1" and variation of the characteristics as follows:

- The name "destra1";

- Position (120,0,0), that is moved from its predecessor, the $120 \mathrm{~cm}$. along the axis "x";

- Optical axis horizontal and parallel to the axis "y";

- Principal distance $63.43 \mathrm{~mm}$.;

- $80 \mathrm{~mm}$ image format. (Horizontal side) $\mathrm{x}$ $60 \mathrm{~mm}$. (Vertical side);

- angle imaging 64.46; 
- Background, as "imageDestra1", the right frame (no. 1988) of the first station (digitized in $800 \times 600$ pixels with 72 dpi resolution), distributed by $10 \mathrm{~mm}$. (0393 inch);

4. connection of two photographic camera in a group called "bicamera1".

\section{Stereometric Modelling}

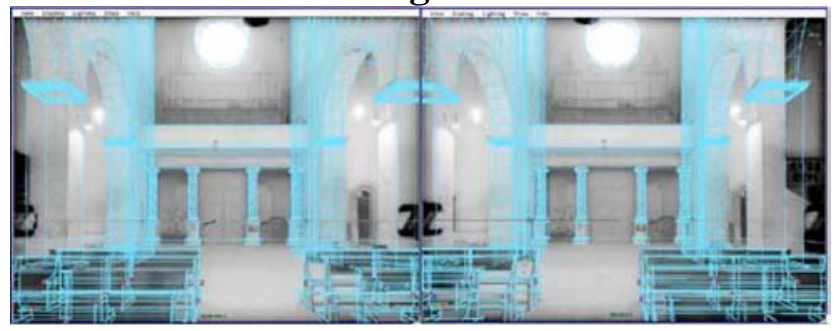

\section{RESULTS}

Thanks to the development of a stereo metric model , either real or virtual, through the "materialization", either real or virtual, of the optical-stereo metric model made visible with a stereoscope, it follows that the photogrammetric survey is used for:

- records purposes, by collecting the data of the photogrammetric survey together with early information about the object and making provision for a file card;

- project purposes, i.e. the design of an

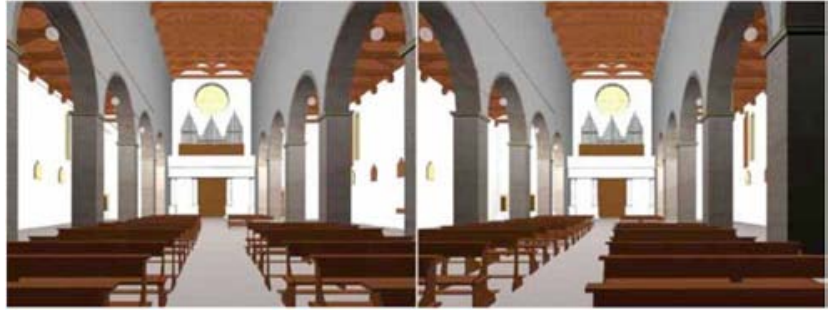

object, either building or furniture. In this case, the pairs of frames are imported into the program and it allows stereo modelling and simultaneous shot of the model taken with a pair of cameras having the same technical features and orientation of the metric cameras used for the photogrammetric survey of the environment;

- restoration purposes, by working on the virtual "reproduction"of the optical-stereometric model imported into the modelling program;

- real reproduction of the surveyed object by means of the virtual reproduction of the imported optical model (using a pair of stereometric photograms) and exportation of the model in CAD-CAM program.

\section{CONCLUSIONS}

It is feasible a continuous on line updating of the cultural heritage with the help of photogrammetry and stereometric modelling. The catalogue of the Architectonic Photogrammetry Laboratory of Politecnico di Bari is available on line at: http://rappresentazione. stereofot. it: 591/StereoFot/FMPro?$d b=$ StereoFot.fp5\&-lay=Scheda $\&-$ format $=$ cerca.htm \&-view

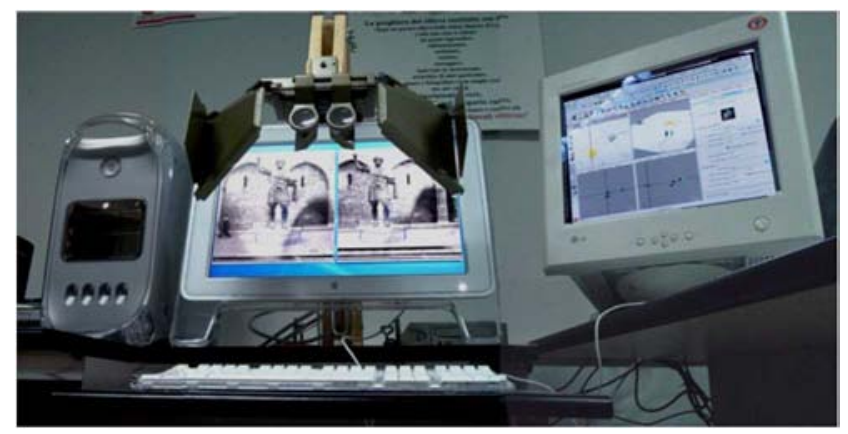

